\title{
A DESTILAÇÃO SIMULAdA NA INDÚSTRIA DO PETRÓLEO
}

\author{
Alexandre de Andrade Ferreira*"\# e Francisco Radler de Aquino Neto \\ Departamento de Química Orgânica, Instituto de Química, Universidade Federal do Rio de Janeiro, Cidade Universitária, CT, \\ Bl. A, 21949-900 Rio de Janeiro - RJ
}

Recebido em 7/6/04; aceito em 15/10/04; publicado na web em 17/2/05

\begin{abstract}
SIMULATED DISTILLATION IN THE PETROLEUM INDUSTRY. One of the most widely used physico-chemical characterizations of hydrocarbon mixtures is the determination of their boiling point distribution. Knowledge of the boiling range of crude oils and petroleum products is essential to ensure the correct specification of final products and to control refinery processes. Simulated distillation, a GC based process, has been playing this role for the past decades in the petroleum industry. The main purpose of this work is to show the fundamentals of this technique as well as its present trends.
\end{abstract}

Keywords: simulated distillation; gas chromatography; ASTM methods.

\section{INTRODUÇÃO}

A palavra "destilação" é originária do latim distillare, que significa "gotejar" e descreve o estágio final do processo, quando do gotejamento de um líquido de um condensador para um recipiente de coleta ${ }^{1}$.

$\mathrm{Na}$ indústria do petróleo, a destilação é o processo de separação mais utilizado. O conhecimento dos dados de distribuição dos pontos de ebulição dos componentes de óleos crus e de produtos do processo de refino do petróleo é essencial para o controle desse processo e a garantia da qualidade do $\mathrm{mesmo}^{2-4}$. Os primeiros procedimentos de destilação, padronizados pela "American Society for Testing and Materials" (ASTM), datam de 1926.

Entretanto, na década de sessenta, foi introduzido o conceito de destilação simulada (DS), que passou a fornecer informações bastante confiáveis para a indústria do petróleo em um intervalo de tempo menor ${ }^{6-9}$. A tecnologia utilizada nesse processo é baseada na cromatografia gasosa, inicialmente proposta com a utilização de colunas recheadas ${ }^{10-13}$. A partir de 1973, a ASTM passou a estabelecer métodos padronizados de análise por destilação simulada: D-3710, D-2887 e D-5307, todos publicados no "ASTM Standards on Chromatography"14. Com o advento da DS, baseada em métodos cromatográficos, os métodos de destilação convencionais passaram a ser denominados métodos de destilação física, como uma forma de diferenciação entre os diferentes processos.

Na DS, o principal objetivo é simular um processo de destilação, como o próprio nome sugere, mas fazendo isso através do uso da cromatografia gasosa. Operado em condições apropriadas, um cromatógrafo a gás funciona como uma unidade de microdestilação altamente eficiente ${ }^{5}$. Revisões sobre o assunto foram publicadas por Butler et al. ${ }^{15}$, Huber ${ }^{16}$, Peaden ${ }^{17} \mathrm{e}$, mais recentemente, por Barman et al. ${ }^{18}$ e Blomberg et al..$^{19}$.

A análise cromatográfica por destilação simulada deve ser capaz de fornecer, como resultado final, uma curva de destilação semelhante à curva gerada pelo método convencional PEV (Pontos

*e-mail: alexandreaf@petrobras.com.br

\# Endereço atual: PETROBRAS, Centro de Pesquisas Leopoldo A. Miguez de Mello, Gerência Geral de Pesquisa e Desenvolvimento de Exploração, Gerência de Geoquímica, Av. Jequitibá, 950, Ilha do Fundão, 21958-900 Rio de Janeiro - RJ de Ebulição Verdadeiros), apresentada na Figura 1. A diferença agora é que essa curva passa a ser obtida em um intervalo de tempo menor, e os valores de PFE (Ponto Final de Ebulição) alcançados são bem mais altos ${ }^{21,22}$.



Figura 1. Curva de destilação obtida pelo método PEV. Adaptada da ref. 20

\section{MÉTODOS DE DS}

Existem cinco métodos utilizados para a destilação simulada, que devem ser escolhidos de acordo com o tipo de amostra que será analisada: D-3710, D-5307 e D-2887, reconhecidos pela $\mathrm{ASTM}^{17}$, e D-2887 estendido e HT-7502,23-25, não reconhecidos pela ASTM, mas bastante utilizados na indústria do petróleo. A Tabela 1 apresenta o tipo de amostra tipicamente analisada por cada método ${ }^{26}$.

A Figura 2 apresenta, para quatro desses métodos, a abrangência com relação ao número máximo de átomos de carbono de compostos passíveis de análise. 
Tabela 1. Métodos de destilação simulada e respectivos tipos de amostra analisados por cada um ${ }^{26}$

\begin{tabular}{|c|c|c|c|c|}
\hline Método & ASTM D 3710 & ASTM D 2887 & ASTM D 5307 & HT 750 \\
\hline Tipo de amostra & $\begin{array}{l}\text { Gasolina } \\
\text { Nafta }\end{array}$ & $\begin{array}{l}\text { Querosene } \\
\text { Diesel } \\
\text { Gasóleo leve } \\
\text { Gasóleo pesado } \\
\text { Neutro leve } \\
\text { Neutro médio } \\
\text { Neutro pesado }\end{array}$ & Petróleo & $\begin{array}{l}\text { Gasóleo pesado } \\
\text { Neutro leve } \\
\text { Neutro médio } \\
\text { Neutro pesado } \\
\text { "Bright stock" } \\
\text { Resíduos }\end{array}$ \\
\hline
\end{tabular}

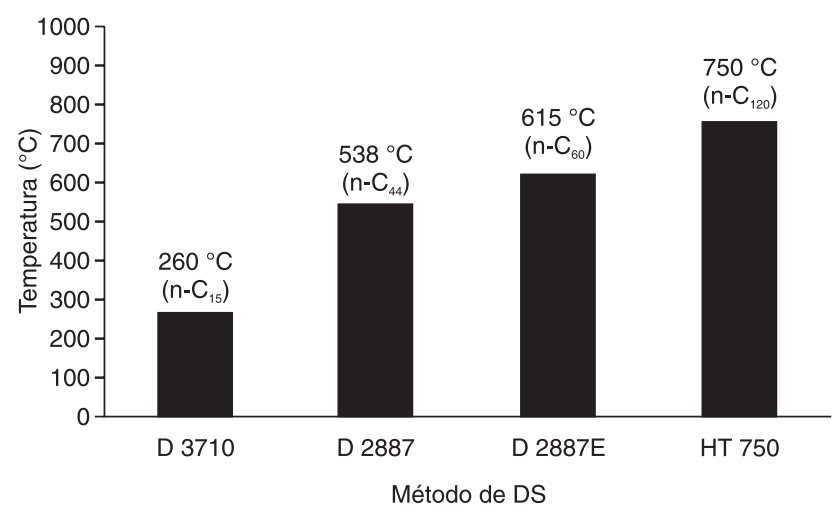

Figura 2. Faixas de abrangência dos métodos de DS em número de átomos de carbono. Adaptada a partir de: SIMDIS Operation \& Maintenance Manual, P/N 59.90.201, versão 2.1, Analytical Controls

\section{COMPARAÇÃO COM OS MÉTODOS DE DESTILAÇÃO FÍSICA}

Para fins de comparação, a Tabela 2 apresenta três dos quatro métodos de destilação física conhecidos (em negrito) e dois dos quatro métodos de destilação simulada citados anteriormente ${ }^{27}$.

Como pode ser visto na Tabela 2, os métodos de DS apresentam melhor reprodutibilidade quando comparados com os métodos de destilação física. Além disso, os tempos de análise das destilações simuladas são bem inferiores: geralmente, em torno de 30 a 50 min. Mas uma das principais vantagens do uso da destilação simulada é, sem dúvida, o alto nível de automação envolvido no processo. Essa automação proporciona não somente a melhor reprodutibilidade observada, como também uma menor intervenção por parte do operador no decorrer da separação ${ }^{27}$. Além disso, há um maior detalhamento composicional da amostra ${ }^{28}$.

\section{FUNDAMENTOS}

A destilação simulada tem como premissa básica o fato de que os componentes da amostra analisada eluem da coluna cromatográfica em ordem crescente de pontos de ebulição ${ }^{2,29,30}$. Isso é normalmente obtido para hidrocarbonetos através do uso de fases estacionárias apolares, como as metil-polisiloxanas e as 5\%-fenil-metilpolisiloxanas ${ }^{17}$. A escolha definitiva da fase está diretamente relacionada ao grau de acordo do resultado da análise com as curvas de destilação reais. Também deve-se atentar para a estabilidade da fase escolhida no que diz respeito às temperaturas máximas de utilização ${ }^{31}$. Dorbon et al. ${ }^{32}$ realizaram um estudo comparativo acerca do uso de alguns tipos de fases estacionárias na análise por destilação simulada. Segundo os autores, as principais divergências surgem quando as amostras analisadas apresentam um elevado índice de compostos aromáticos. Esse é o caso das frações geradas em processos de craqueamento catalítico fluido e craqueamento térmico, onde o teor de aromáticos pode chegar a $80 \%{ }^{32}$.

Porém, antes da injeção da amostra, o sistema cromatográfico deve ser calibrado e avaliado quantitativamente. A seqüência de calibração incluii ${ }^{23,33}$ : a) uma análise em branco (com solvente ou não); b) uma análise de uma mistura de parafinas de cadeia normal com pontos de ebulição bem conhecidos e c) uma análise de uma amostra de referência.

O cromatograma gerado pela análise em branco, a primeira dessa sequiência, é subtraído dos cromatogramas da amostra de forma a eliminar fatores como impurezas do gás de arraste, ruído provocado pelo detector, sangramento da coluna e impurezas do septo ${ }^{23}$. As primeiras publicações sobre o assunto surgiram no final da década de 70 e início da de $80^{29,34,35}$. São basicamente três os métodos utilizados para a compensação de linha de base a partir da análise em branco ${ }^{29,35}$ : subtração ponto a ponto de uma linha de base armazenada; subtração ponto a ponto após zerar matematicamente a linha de base inicial com a armazenada e reconstrução de uma linha de base armazenada, de forma que os pontos do início e do final sejam os mesmos do cromatograma da amostra.

A análise da mistura de parafinas (segunda análise da seqüência de calibração), por sua vez, permite a construção de uma curva de calibração que relaciona pontos de ebulição e tempos de retenção. A partir dessa curva e com base na avaliação do resultado da amostra de referência (analisada em seguida), as amostras reais são injetadas e as curvas de destilação são obtidas para cada uma delas $^{23}$. Exemplos da análise de uma mistura de n-parafinas, a curva de calibração correspondente e a análise de uma amostra de referência são apresentados nas Figuras 3, 4 e 5, respectivamente.

No que diz respeito ao detector, sua resposta deve ser relacionada à massa ou ao volume dos componentes da amostra, o que é

Tabela 2. Comparação de algumas características de métodos de destilação física (células em negrito) e destilação simulada ${ }^{27}$

\begin{tabular}{lcccc}
\hline Método & Ponto Final de Ebulição $\left({ }^{\circ} \mathrm{C}\right)$ & Tempo de Análise $(\mathrm{h})$ & Calibração & Reprodutibilidade $\left({ }^{\circ} \mathrm{C}\right)$ \\
\hline D-2892 & 370 & 20 & manual & 30 \\
D-86 & 370 & 1 & manual & 10 \\
D-1160 & 550 & 3 & manual & 20 \\
D-3710 & 260 & 0,5 & automática & 3 \\
D-2887 & 538 & 0,5 & automática & 2 \\
\hline
\end{tabular}






Figura 3. Análise cromatográfica de uma mistura de parafinas de cadeia normal (amostra de calibração SIMDIS HT-750, P/N 59.50.101A $\left(C_{5}-C_{28}\right)$, $59.50 .100 B\left(C_{30}-C_{120}\right)$, Analytical Controls). Gráfico do sinal versus tempo de retenção (min) e temperatura de ebulição $\left({ }^{\circ} \mathrm{C}\right)$

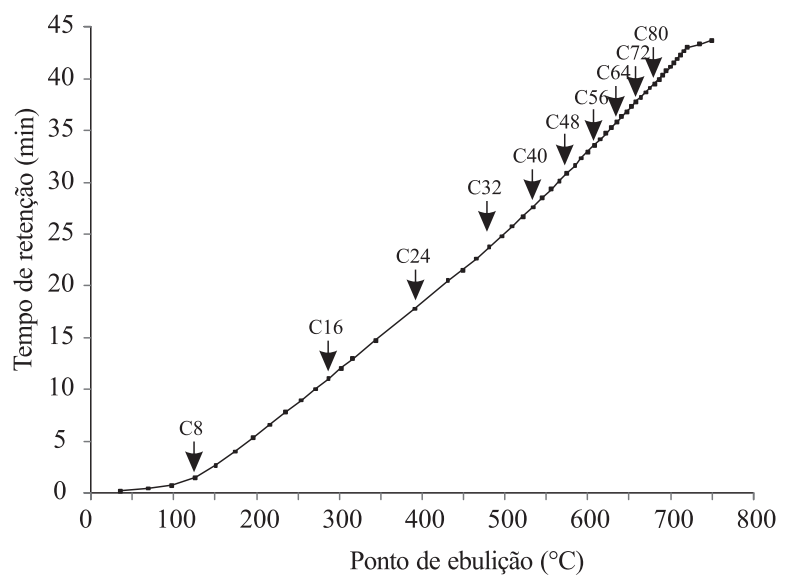

Figura 4. Exemplo de curva de calibração construída com base na análise de mistura de parafinas de cadeia normal (obtida com o software SIMDIS, P/N 80109.3XX, versão 04.10, Analytical Controls). Gráfico do tempo de retenção (min) versus temperatura de ebulição $\left({ }^{\circ} \mathrm{C}\right)$

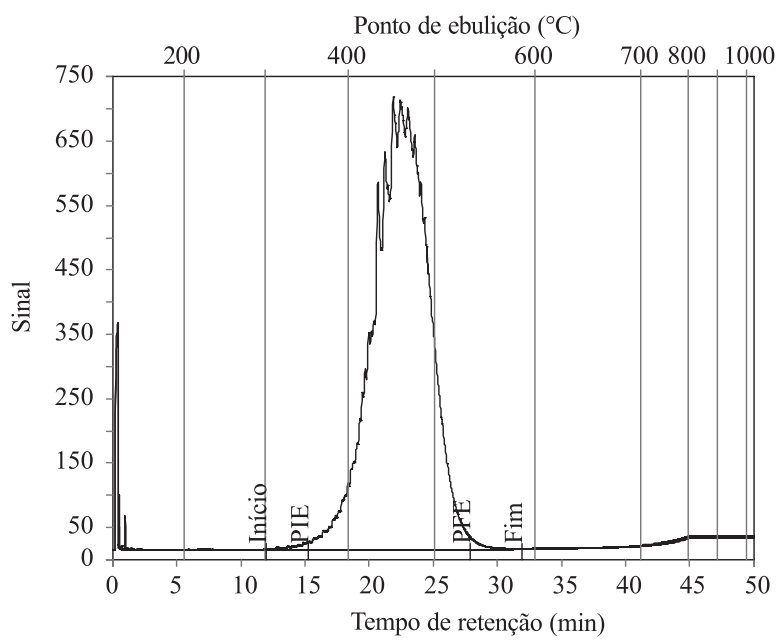

Figura 5. Análise cromatográfica de uma amostra de referência (amostra de referência SIMDIS HT-750, P/N 56.40.202, Analytical Controls). Gráfico do sinal versus tempo de retenção ( $\mathrm{min})$ e temperatura de ebulição $\left({ }^{\circ} \mathrm{C}\right)$ feito através do uso de "softwares" específicos. Os dois detectores mais comumente utilizados são o detector por condutividade térmica (DCT) e o detector por ionização em chama (DIC) $)^{17}$.

Com relação à introdução de amostra, os injetores preferencialmente utilizados para as análises por DS são o injetor na coluna a frio ${ }^{13,22,36}$ e o injetor por vaporização com temperatura programável ${ }^{23,24,37,38}$, já que os mesmos diminuem significativamente a discriminação, na agulha, de componentes mais pesados, na transferência da amostra da seringa para o cromatógrafo. Entretanto, o injetor com e sem divisão de fluxo já foi empregado em alguns estudos mais específicos ${ }^{39,40-42}$.

\section{COLUNAS RECHEADAS X COLUNAS CAPILARES}

Conforme já descrito anteriormente, o primeiro método ASTM utilizado para a análise por destilação simulada (D-2887, implantado em 1973) admitia o uso de colunas recheadas. O limite prático de temperatura da maior parte dessas colunas é de $350{ }^{\circ} \mathrm{C}$. A essa temperatura, o $\mathrm{n}-\mathrm{C}_{44}\left(\mathrm{PE}=545^{\circ} \mathrm{C}\right)$ é a parafina mais pesada que elui normalmente nessa $\mathrm{DS}^{22}$, o que limita as análises com essas colunas a esse número aproximado de átomos de carbono (44) dos compostos passíveis de análise. Além dessa desvantagem, as colunas recheadas também apresentam um elevado sangramento da fase estacionária a temperaturas elevadas - tornando a compensação de linha de base mais difícil - e retêm mais os componentes de uma amostra em comparação com uma coluna capilar semelhante ${ }^{17}$. Mas essas colunas se mantêm até hoje na rotina de análises desse tipo. Os que defendem seu uso alegam que as mesmas acomodam grandes volumes de amostra injetada, em comparação com as colunas capilares. Além disso, a grande capacidade de retenção dessas colunas as tornaria mais adequadas para as amostras com componentes de baixo ponto de ebulição, sem o emprego de resfriamento criogênico ${ }^{17}$. Entretanto, ocorre que, no primeiro caso, a injeção de um volume maior de amostra é compensada, na coluna capilar, por uma maior resolução dos picos cromatográficos devido ao fato de estes serem bem mais finos, o que aumenta muito a razão sinal/ruído (compensando largamente o menor volume injetado). No que diz respeito à retenção dos componentes mais leves, bastaria a utilização de uma coluna capilar de fase espessa.

O emprego das colunas capilares nas análises cromatográficas por destilação simulada teve início na década de 70, com Jackson et al $^{43}$. Na ocasião, os autores utilizaram uma coluna de aço inox de $10 \mathrm{~m}$ de comprimento com 0,01 polegada de diâmetro interno para a obtenção de curvas de destilação para três petróleos diferentes. Concluiu-se que o uso da coluna tubular aberta permitia a determinação acurada e rápida dessas curvas de distribuição de faixas de ebulição.

A partir de então, uma série de estudos empregando colunas capilares distintas (e aqui estão incluídas as colunas "megabore", com $0,53 \mathrm{~mm}$ de diâmetro interno) foi confirmando a maior eficiência das mesmas em relação às colunas recheadas. Luke e Ray ${ }^{44}$, por exemplo, utilizaram colunas de vidro de $0,32 \mathrm{~mm}$ de diâmetro interno na análise de resíduos atmosféricos, chegando a um PFE de $650{ }^{\circ} \mathrm{C}$ (o que corresponde à temperatura de eluição do $\mathrm{C}_{60}$ ).

Em trabalho posterior, Firor $^{45}$, em um dos estudos pioneiros no uso sistemático das colunas capilares para destilação simulada, enumerou uma série de vantagens no emprego das mesmas: a) maior estabilidade da FE e conseqüente aumento no tempo de vida útil da coluna; b) tempo de análise reduzido; c) eluição de frações de petróleo com PFE acima de $538{ }^{\circ} \mathrm{C}$; d) compatibilidade com injeção na coluna a frio automatizada e e) maior repetitividade.

Essas mesmas conclusões também foram tiradas, em maior ou menor extensão, nos trabalhos que se seguiram ${ }^{10,12,31,36,46-49}$, conso- 
lidando definitivamente o uso das colunas capilares na análise por destilação simulada.

Atualmente, com o desenvolvimento de novos materiais para as colunas, de fases estacionárias de baixo sangramento e da capacidade de programação do forno dos cromatógrafos com repetitividade para alcance de $430{ }^{\circ} \mathrm{C}$ ou mais, tornou-se possível a eluição de hidrocarbonetos normais contendo até 140 átomos de carbono, a uma resolução maior que a exigida pelo método ASTM $^{11,30,35,36,50-54}$. Seguindo esta tendência, a "American Society for Testing and Materials" publicou recentemente a norma D-6352, que contempla a determinação da faixa de ebulição de frações de petróleo de 174 a $700{ }^{\circ} \mathrm{C}$, abrangendo os hidrocarbonetos normais contendo de 10 a 90 átomos de carbono ${ }^{55}$.

\section{EXPRESSÃO DOS RESULTADOS}

Os dados obtidos da destilação simulada podem ser relatados, basicamente, de três formas: o cromatograma em si, uma curva de área percentual versus temperatura; e uma tabela contendo as informações contidas nessa curva. A Figura 6 mostra um exemplo de cromatograma obtido por destilação simulada empregando uma

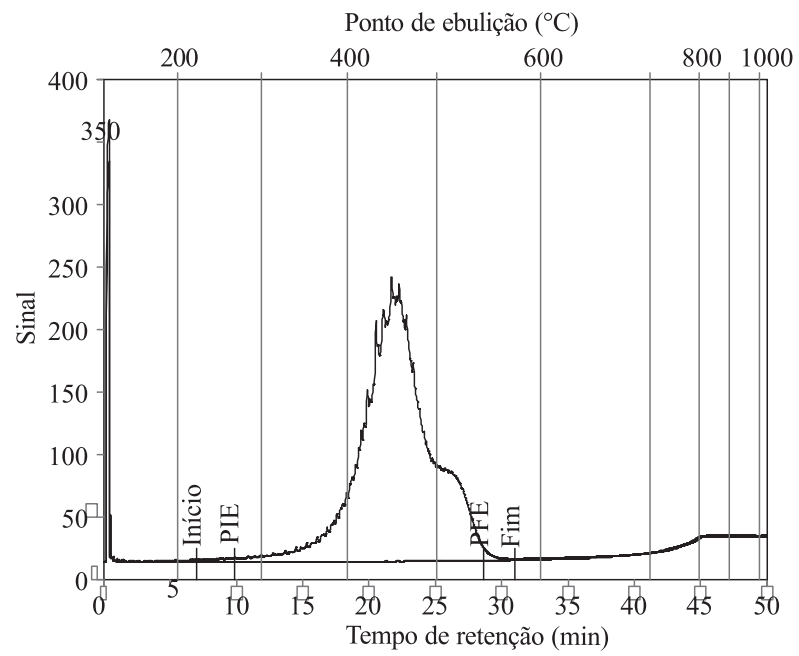

Figura 6. Exemplo de um cromatograma típico utilizando uma coluna capilar curta. Gráfico do sinal versus tempo de retenção (min) e temperatura de ebulição $\left({ }^{\circ} \mathrm{C}\right)$

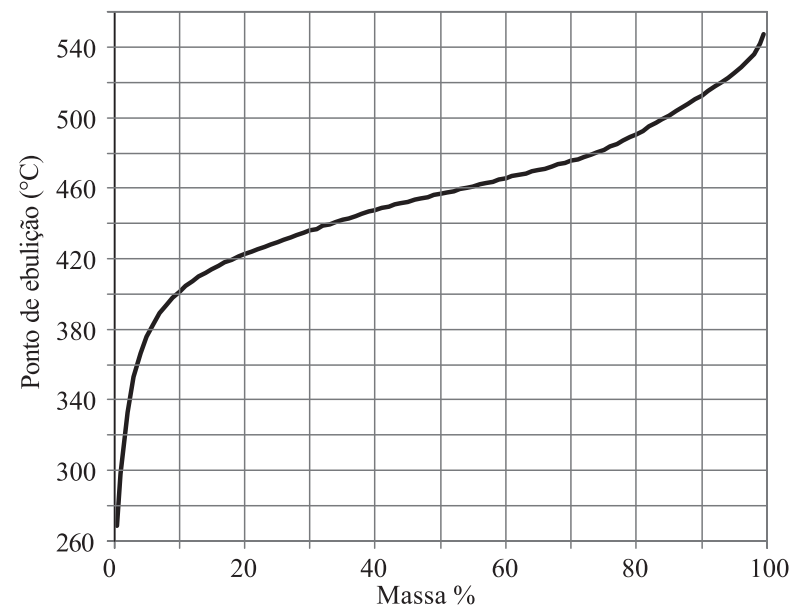

Figura 7. Exemplo de uma curva de temperatura de ebulição dos componentes da mistura versus massa percentual acumulada (obtida com o software SIMDIS, P/N 80109.3XX, versão 04.10, Analytical Controls)
Distribuição de pontos de ebulição (PE)

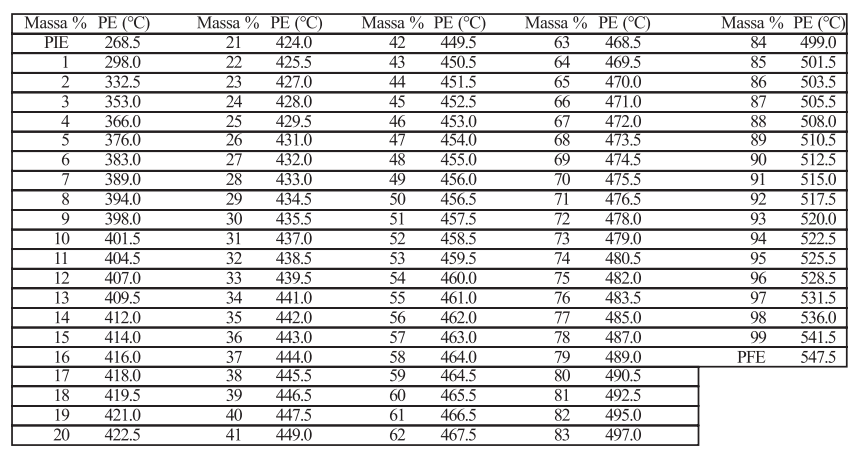

Figura 8. Exemplo de um relatório emitido considerando o método ASTM D 2887 (obtido com o software SIMDIS, P/N 80109.3XX, versão 04.10, Analytical Controls)

coluna capilar curta. A Figura 7 apresenta o resultado da mesma análise na forma do gráfico "área percentual versus temperatura". A Figura 8 mostra um relatório com as informações contidas na curva da Figura 6. Este relatório pode ser maior que o apresentado aqui, podendo, inclusive, ser personalizado, mostrando as faixas de corte de interesse e com incrementos de 5 em 5 ou de 10 em $10{ }^{\circ} \mathrm{C}$, por exemplo.

\section{AGRADECIMENTOS}

À Gerência de Engenharia e Operação de Plantas Piloto do CENPES/PETROBRAS pelos comatogramas e dados fornecidos (Figuras 3 a 8), e em especial pelo apoio dos seus técnicos químicos (Laboratório de Apoio Analítico Específico). Agradecimentos também à química de petróleo F. R. D. Faria, da Gerência de Química do CENPES/PETROBRAS, pelas informações e discussões imprescindíveis para a realização deste trabalho, e ao grupo de Desenho da Gerência de Geoquímica do CENPES/PETROBRAS pela arte final das figuras apresentadas.

\section{REFERÊNCIAS}

1. Karasek, F. W.; Res. Develop. 1969, 69, 68

2. Grutterink, D.; Nota de aplicação HP/AC 1995a, 228.

3. Dong, D.; Liu, Y.; Tang, J.; Pet. Sci. Technol. 1999, 17, 209.

4. Ukwuoma, O.; Pet. Sci. Technol. 2002, 20, 525.

5. Schwartz, H. E.; Robert, G. B.; Mieczyslaw, M. B.; Fu, S.; Anal. Chem. 1987, 59, 1393

6. Eggertsen, F. T.; Groennings, S.; Holst, J. J.; Anal. Chem. 1960, 32, 904.

7. Green, L. E.; Schmauch, L. J.; Worman, J. C.; Anal. Chem. 1964, 36, 1512.

8. Petrocelli, J. A.; Puzniak, T. J.; Clark, R. O.; Anal. Chem. 1964, 36, 1008.

9. Roussis, S. G.; Fitzgerald, W.P.; Anal. Chem. 2000, 72, 1400.

10. Noel, F.; J. High Resoln. Chromatogr. Chromatogr. Commun. 1988, 11, 837.

11. Thomson, J. S.; Rynaski, A. F.; J. High Resoln. Chromatogr. 1992, 15, 227.

12. Workman, D. S.; Noel, F.; Watt, M. R.; J. Chromatogr. Sci. 1993, 31, 95.

13. Bacaud, R.; Rouleau, L.; J. Chromatogr. A 1996, 750, 97.

14. ASTM Standards on Chromatography, American Society for Testing and Materials, Mars, Pennsylvania, USA, 1989; D-2887 p. 216, D-3710 p. 441, D-5307 p. 564, Proposed Test Method for Boiling Range Distribution of Crude Petroleum by Gas Chromatography p. 771, Proposed Test Method for Carbon Number Distribution of Paraffin Wax by Gas Chromatography p.778, Proposed Test Method for Carbon Number Distribution of Crude Petroleum, p. 790.

15. Petroff, N.; Hoscheitt, A.; Durand, J. P.; J. Chromatogr., A 1987, 395, 241.

16. Zuber, K.; Bartl, P.; Fuel 1989, 68, 659.

17. Peaden, P. A.; J. High Resoln. Chromatogr. 1994, 17, 203.

18. Barman, B. N.; Cebolla, V. L.; Membrado, L.; Crit. Rev. Anal. Chem. 2000, 30, 75 .

19. Blomberg, J.; Schoemakers, P. J.; Brinkman, U. A. Th.; J. Chromatogr., A 2002, 972, 137. 
20. Farah, M. A. Em Petróleo e Derivados I-Caracterização do Petróleo e Seus Produtos; Programa Trainees PETROBRAS, 2002, p. 38.

21. Shariff, S. M.; Tong, D.; Bartle, K. D.; J. Chromatogr. Sci. 1994, 32, 541.

22. Neer, L. A.; Deo, M. D.; J. Chromatogr. Sci. 1995, 33, 133.

23. Grutterink, D.; Nota de aplicação HP/AC 1995b, 228.

24. Curvers, J.; Van Den Engel, P.; J. High Resoln. Chromatogr. 1989, 12, 16.

25. Faria, F. R. D.; comunicação técnica 161/96, CENPES/PETROBRAS, 1996.

26. Faria, F. R. D.; comunicação pessoal.

27. HP/AC; Nota técnica $\mathbf{1 9 9 6 .}$

28. Spieksma, W.; J. Chromatogr. Sci. 1998, 36, 467.

29. Abbott, D. J.; J. Chromatogr. Sci. 1983, 21, 425.

30. Durand, J. P.; Bré, A.; Bébouléne, J. J.; Ducrozet, A.; Carbonneaux, S.; J. Chromatogr. Sci. 1998, 36, 431.

31. Durand, J. P.; Analusis 1998, 26, M17.

32. Dorbon, M.; Lamaison, S.; Chevalier, A.; J. Chromatogr. 1991, 557, 155.

33. Anderson, G.; Nota de aplicação HP 1986, LDS 89-1.

34. Southern, T. G.; Iacchelli, A.; Cuthiell, D.; Selucky, M. L.; Anal. Chem. 1985, 57, 303

35. Trestianu, S.; Zilioli, G.; Sironi, A.; Saravelle, C.; Munari, F.; Galli, M.; Gaspar, G.; Colin, J.M.; Jovelin, J. L.; J. High Resoln. Chromatogr. Chromatogr. Commun. 1985, 8, 771.

36. Firor, R. L.; Phillips, R. J.; J. High Resoln. Chromatogr. 1989, 12, 181.

37. Disanzo, F. P.; Lowther, C. J.; Yoder, R. E.; Lane, J. L.; J. Chromatogr. Sci. 1988, 26, 39.

38. Lubkowitz, J. A.; Meneghini, R. I.; J. Chromatogr. Sci. 2002, 40, 269.

39. Schomburg, G.; Häusig, U.; J. High Resoln. Chromatogr. Chromatogr. Commun. 1985, 8, 572 .

40. Giarrocco, V.; Nota de aplicação HP 1997a, 228.
41. Giarrocco, V.; Nota de aplicação HP 1997b, 228.

42. Quimby, B. D.; Grudoski, D. A.; Giarrocco, V.; J. Chromatogr. Sci. 1998 36,435 .

43. Jackson, B. W.; Judges, R. W.; Powell, J. L.; J. Chromatogr. Sci. 1976, 14 49.

44. Luke, L. A.; Ray, J. E.; J. High Resoln. Chromatogr. Chromatogr. Commun. 1985, 8, 193.

45. Firor, R.L.; Nota de aplicação HP 228-60 1988.

46. Rastogi, A.; J. High Resoln. Chromatogr. Chromatogr. Commun. 1987, 10 , 477.

47. Firor, R. L.; Am. Lab. 1989, 21, 32.

48. Zou, N.; Yusheng, T.; Jiahe, S.; Wanzhen, L.; J. High Resoln. Chromatogr. Chromatogr. Commun. 1993, 16, 188.

49. Bacaud, R.; Rouleau, L.; Cebolla, V. L.; Membrado, L.; Vela, J.; Catal. Today 1998, 43, 171 .

50. Lipsky, S. R.; Duffy, M.L.; J. High Resoln. Chromatogr. Chromatogr. Commun. 1986, 9, 376

51. Termonia, M.; Munari, F.; Sandra, P.; J. High Resoln. Chromatogr. Chromatogr. Commun. 1987, 10, 263

52. Reddy, K. M.; Wei, B.; Song, C.; Catal. Today 1998, 43, 187.

53. Sharma, B. K.; Sarowha, S. L. S.; Bhagat, S. D.; J. Sep. Sci. 2003, 26, 1657.

54. Raia, J. C.; Villalanti, D. C.; Subramanian, M.; Williams, B.; J. Chromatogr. Sci. 2000, 38, 1 .

55. American Society for Testing and Materials, Mars, Pennsylvania, USA, 2003; D-6352-03, Standard Test Method for Boiling Range Distribution of Petroleum Distillates in Boiling Range from 174 to $700{ }^{\circ} \mathrm{C}$ by Gas Chromatography. 